\title{
ArasBeam: when amateurs contribute to Be star research
}

\author{
François Cochard ${ }^{1,2}$, Valérie Desnoux ${ }^{2}$ and Christian Buil ${ }^{2}$ \\ ${ }^{1}$ Shelyak Instruments, France - http://www.shelyak.com \\ email: francois.cochard@shelyak.com \\ ${ }^{2}$ Aras, Amateur Ring for Astronomical Spectroscopy, http://astrosurf.com/aras \\ email: valerie.desnoux@free.fr, christian.buil@wanadoo.fr
}

\begin{abstract}
Since 2003, the amateur astronomical community has decided, in collaboration with the Paris-Meudon Observatory, to coordinate their observations to get the best spectral survey of Be stars as possible. A database for amateur and professional Be star spectra, BeSS, has been created. Spectrographs (up to $\mathrm{R}=20000$ ) and software tools have been developed for amateurs. Among them, ArasBeam is a web-based tool designed to organize amateur Be spectral observations. A very simple color coding indicates to any observer which stars must be observed on the following night to get the best possible survey of Be stars. So far, more than 11000 amateur spectra have been collected in BeSS. About all bright Be stars (up to magnitude 8) listed in BeSS and visible from the Northern hemisphere have been observed at least one time. In addition, 6 outbursts have been detected by amateurs in the last 2 years.
\end{abstract}

Keywords. stars: emission-line, Be, techniques: spectroscopic, stars: activity, surveys

\section{Amateurs, spectroscopy and Be stars}

Be stars are easy targets for small instruments (300 stars up to magnitude 8, Northern hempisphere), their spectra evolve on different timescales (from days to years), periodically or with episodic outbursts. These stars require a continuous observation, to better understand the Be phenomenon.

Amateur spectroscopists are numerous, dispatched over the world, and available very quickly. They can provide observations that are complementary to professional observations. The amateur community has developed several tools to contribute to Be spectroscopic observations :

- spectrographs, such as Lhires III or eShel (see Thizy et al., these proceedings),

- software for spectra processing (Iris, Vspec, Audela,...),

- a mailing list: Spectro-l (http://groups.yahoo.com/group/spectro-l),

- and ArasBeam, a web-based tool to coordinate Be observations.

Our spectra are uploaded in the BeSS database (see de Batz et al., these proceedings). The observing program is simple: we focus on $\mathrm{H} \alpha$, observe all Be stars up to $\mathrm{V}$ magnitude 8 at least once a year, detect as many outbursts as possible, and track them intensively.

\section{The tool}

Arasbeam is available at $h t t p: / /$ arasbeam.free.fr/?lang=en. ArasBeam is a website developed and managed by amateurs. It allows to show the list of Be stars from BeSS catalog with dynamic sorting keys and display all existing BeSS spectra for a given Be star. For each Be star an ideal observing period is defined depending on its activity. By default, this period is one year. In this way, we provide priorities for observations, 

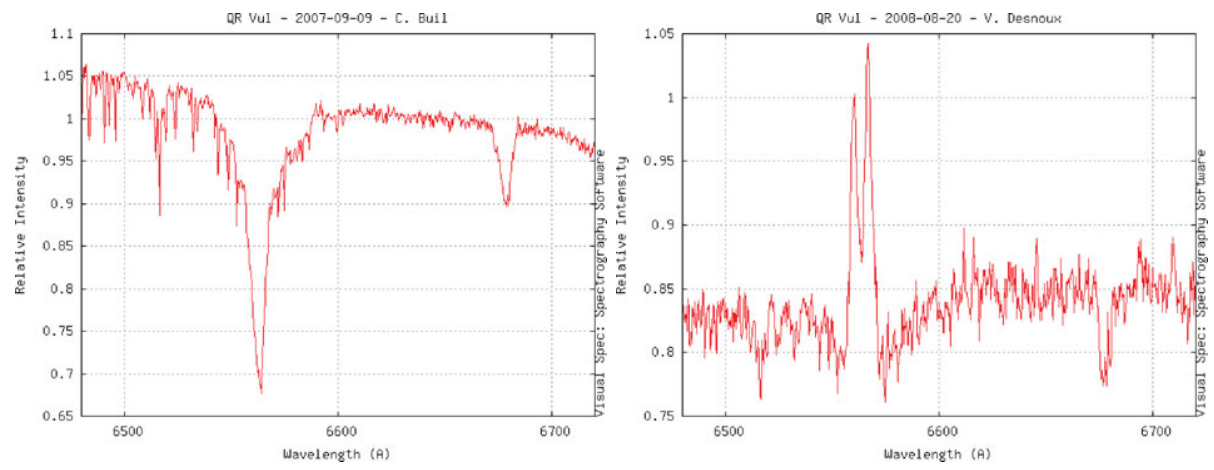

Figure 1. QR Vul, before and after the outburst discovery in Aug. 2008.

based on ideal periods and the latest observations. This is given with a visual color coding. ArasBeam also allows to distribute news on Be observations via a multi-authors platform. Finally, ArasBeam is meant to coordinate amateur observations: it is when amateurs work together that they become really complementary to professionnals.

\section{Current results}

- 31 amateur observers recorded in BeSS (mainly in the Northern hemisphere);

- More than 11000 amateur spectra have been uploaded in BeSS so far. 3000 of them are at $\mathrm{H} \alpha$;

- 295 Be stars up to V magnitude 8 have at least one spectra. This covers $97 \%$ of the BeSS catalog up to $\mathrm{V}=8$ for the Northern hemisphere;

- 6 outbursts have been detected in 3 years of observations: QR Vul (see Fig.1), HD 22780, $\lambda$ Cyg, $\lambda$ Eri, HD 37149, and HD 34959;

- 5 other Be stars, known as higly active, have a high observation rate: $\theta \mathrm{CrB}, 66 \mathrm{Oph}$, V1040 Sco, and HD 57682;

- 17 stars have more than 10 spectra obtained during the last year.

\section{Next steps}

In the future, we plan to increase the number of observers and observations, by communication, training, and improving tools. We also plan to analyze the current data to classify stars depending on their activity. Based on these data, optimize the ideal observing period for each Be star. The goal is to increase the number of outburst detections.

\section{Conclusion}

Spectrography is quite new to amateur astronomers. However, this activity is growing fast and is now mature. The data we have collected during the last years are very complementary to professionnal data: we are limited in magnitude and signal-to-noise ratio, but we have a much better time coverage, for a high number of stars. Knowing that about $80 \%$ of professionnal observations are obtained in spectroscopy, we can easily understand that when amateurs start working in this area, this opens a lot of opportunity for new Professionals/Amateurs collaborations. The Be star observing program is a perfect example of such a succesful collaboration. This program is possible thanks to an active work on both sides, amateur and professionnal. 